\title{
Wolbachia requirement for oogenesis: occurrence within the genus Asobara (Hymenoptera, Braconidae) and evidence for intraspecific variation in $A$. tabida
}

\author{
F Dedeine, $\mathrm{M}$ Boulétreau and $\mathrm{F}$ Vavre \\ Biométrie et Biologie Evolutive, UMR-CNRS 5558, Université Lyon 1, 69622 Villeurbanne cédex, France
}

\begin{abstract}
Wolbachia are symbiotic bacteria that induce a diversity of phenotypes on their numerous invertebrate host species. In the wasp Asobara tabida (Braconidae), each individual harbours three Wolbachia strains: wAtab3, which is required for host oogenesis, and wAtab1 and wAtab2, that do not have this function but induce cytoplasmic incompatibility. In this study, we surveyed and identified Wolbachia strains in four additional Asobara species. We detected Wolbachia in one of these species, but both the identity (based on wsp gene) and prevalence of the Wolbachia detected in natural population indicate that this host species is not dependent on Wolbachia for oogenesis. We also compared $A$. tabida lines of different geographical origin for their dependence on Wolbachia. All individuals from $16 \mathrm{~A}$. tabida lines proved to be infected by the three Wolbachia strains wAtab1, wAtab2 and wAtab3, but, interestingly, we found variation among
\end{abstract}

lines in the degree to which females were dependent on Wolbachia to produce their oocytes. In three lines, aposymbiotic females (cured from the three Wolbachia strains by antibiotics) can produce some oocytes. However, these aposymbiotic females produce fewer and smaller oocytes than symbiotic ones, and the larvae they produce die before full development. Thus, depending on which nuclear genotype they have, $A$. tabida females depend on Wolbachia either because they fail to produce any oocyte or because the few oocytes they do produce generate unviable offspring. We discuss the implications of these findings for the understanding of the physiological and genetic deficiency of aposymbiotic females.

Heredity (2005) 95, 394-400. doi:10.1038/sj.hdy.6800739; published online 24 August 2005

Keywords: Asobara tabida; Drosophila parasitoid; insect development; oogenesis; symbiosis; Wolbachia

\section{Introduction}

Wolbachia form a monophyletic group of alpha-proteobacteria that infect many arthropods and filarial nematodes. Living within the cells of their host, they are maternally transmitted from mother to offspring through the egg cytoplasm (Werren, 1997; Bandi et al, 1998; Stouthamer et al, 1999). Wolbachia have received much attention for three main reasons. First, several surveys suggest that about $20 \%$ of all insect species may be infected with Wolbachia, making these bacteria one of the most widespread parasites yet identified (Werren et al, 1995; West et al, 1998; Werren and Windsor, 2000). Second, Wolbachia have proved to be masters of modifying arthropod reproduction, including cytoplasmic incompatibility, parthenogenesis, male-killing or feminization. All these modifications allow the infection to spread and persist within host populations (Werren, 1997; Stouthamer et al, 1999). Third, in addition to these immediate reproductive modifications, Wolbachia infection may have a range of long-term effects on host

Correspondence: F Dedeine, Department of Entomology, University of Wisconsin-Madison, 643 Russell Labs, 1630 Linden Drive, Madison, WI 53706, USA. E-mail: fdedeine@entomology.wisc.edu

Received 8 December 2004; accepted 15 July 2005; published online 24 August 2005 taxa, such as providing a possible mechanism of rapid speciation (Bordenstein, 2003) or influencing the evolution of sex-determining mechanisms (Rigaud, 1997).

In the great majority of arthropods, Wolbachia are facultative for host physiology: individuals cured of the infection remain physiologically functional and able to feed and reproduce. In contrast to this rule, however, some species have become completely dependent on Wolbachia infection for reproduction (reviewed in Gottlieb and Zchori-Fein, 2001; Dedeine et al, 2003). One of these exceptions is the Drosophila parasitoid wasp Asobara tabida (Hymenoptera, Braconidae), in which females completely depend on Wolbachia infection for oogenesis. Aposymbiotic individuals (antibiotic-cured) of both sexes have an apparently normal morphology, physiology and behaviour, except that females have empty ovaries and fail to produce any oocyte (Dedeine et al, 2001). The possibility that the specific inhibition of oocyte production is caused directly by antibiotics, or indirectly through the release of endotoxins from decaying bacteria, has been rejected, thus strongly suggesting that oogenesis is totally dependent on infection itself (Dedeine et al, 2001). More recently, it has been demonstrated that, of the three genotypic Wolbachia strains co-infecting each $A$. tabida individual, only one strain, wAtab3, is required for oogenesis, whereas the two others, wAtab1 and wAtab2, do not have this ability, but induce cytoplasmic incompatibility (Dedeine et al, 2004). 
How oogenesis has become dependent on wAtab3 in the course of evolution of this insect lineage remains an open question. However, a previous result has suggested that this event occurred recently in the Asobara lineage (Dedeine et al, 2001): another species of the same genus, A. citri, does not harbour any Wolbachia, suggesting that the dependence on wAtab3 has evolved after the divergence of these two Asobara species. The aim of this study was to test whether the dependence on wAtab3 has emerged recently in Asobara lineage by studying the prevalence, identity and role of Wolbachia in oogenesis both in different species of the genus Asobara and within the species $A$. tabida. We confirmed preliminary results from $A$. citri, and surveyed the presence and identity of Wolbachia infections in three other Asobara species: A. rufescens, a very closely related species to $A$. tabida (Vet and Janse, 1984; Vet et al, 1984), and two more divergent species, A. persimilis from Australia, and $A$. sp, an undescribed species from North America. We also looked for intraspecific variation in the degree of wAtab3 dependence of oocyte production within $A$. tabida. To accomplish this, we compared Wolbachia infection and their role in oocyte production in 16 laboratory lines of A. tabida established from foundresses obtained from different areas through Europe and North America. In three lines, we found that aposymbiotic females were able to produce some oocytes. We then characterized these oocytes (number and size), assessed whether females could deposit them into Drosophila host larvae and tested whether aposymbiotic eggs are able to develop into fertile adults.

\section{Materials and methods}

\section{Biological material}

Wasps of the genus Asobara (Hymenoptera, Braconidae: Alysiinae) are solitary endoparasitoids of several Drosophila species and related genera. Females lay their eggs in fly larvae, within which parasitic larvae feed and develop (Carton et al, 1986). In this work, we studied five Asobara species from 25 localities of four continents (Table 1). Phylogenetic relationships between these species have not been established so far, but several morphological, genetic and ecological studies clearly indicate that $A$. rufescens is the closest related species to A. tabida (Vet and Janse, 1984; Vet et al, 1984). Except for $A$. rufescens, all the wasps were from established laboratory lines. Parasitoids were reared on a Wolbachiafree D. melanogaster strain originating from St Foy-lès-Lyon (Rhône, France). Rearing was conducted in vials containing standard Drosophila diet (David, 1962), at $20^{\circ} \mathrm{C}$ under a 12:12 light:dark cycle and $70 \% \mathrm{RH}$. A. rufescens wasps are known to develop on another host species, Scaptomyza pallida, which is more difficult to maintain in laboratory than $D$. melanogaster. For this reason, all $A$. rufescens individuals studied here were collected from the field.

\section{Wolbachia detection and identification}

To detect Wolbachia infection by PCR, we used a set of internal primers specific for the Wolbachia ftsZ gene (for primer sequences, see Dedeine et al, 2001). ITS 2 primers

Table 1 Presence and identity of Wolbachia in the Asobara wasps studied

\begin{tabular}{|c|c|c|c|c|c|}
\hline Species & Locality & Country & $\begin{array}{l}\text { Individuals tested } \\
\text { (no. males-females) }\end{array}$ & $\begin{array}{l}\text { Proportion of } \\
\text { infection }\end{array}$ & Wolbachia strains ${ }^{\mathrm{a}}$ \\
\hline A. tabida & $\begin{array}{l}\text { Cordes } \\
\text { Evans } \\
\text { Lablachère } \\
\text { Malaucène } \\
\text { Pierrefeu } \\
\text { Plascassier } \\
\text { Sospel } \\
\text { St Foy-lès-Lyon } \\
\text { St Laurent } \\
\text { Villette } \\
\text { Wervicq-sud } \\
\text { Hoge veluwe } \\
\text { Leiden } \\
\text { Kos } \\
\text { Saanich } \\
\text { Seattle }\end{array}$ & $\begin{array}{l}\text { France } \\
\text { France } \\
\text { France } \\
\text { France } \\
\text { France } \\
\text { France } \\
\text { France } \\
\text { France } \\
\text { France } \\
\text { France } \\
\text { France } \\
\text { The Netherlands } \\
\text { The Netherlands } \\
\text { Greece } \\
\text { Canada } \\
\text { United States }\end{array}$ & $\begin{array}{c}5-5 \\
5-7 \\
4-6 \\
5-5 \\
12-32 \\
5-5 \\
5-5 \\
5-5 \\
5-5 \\
5-5 \\
5-5 \\
6-8 \\
8-9 \\
5-5 \\
7-10 \\
6-9\end{array}$ & $\begin{array}{l}1 \\
1 \\
1 \\
1 \\
1 \\
1 \\
1 \\
1 \\
1 \\
1 \\
1 \\
1 \\
1 \\
1 \\
1 \\
1\end{array}$ & $\begin{array}{l}1,2,3 \\
1,2,3 \\
1,2,3 \\
1,2,3 \\
1,2,3^{\mathrm{b}} \\
1,2,3 \\
1,2,3 \\
1,2,3 \\
1,2,3 \\
1,2,3 \\
1,2,3^{\mathrm{b}} \\
1,2,3 \\
1,2,3^{\mathrm{b}} \\
1,2,3^{\mathrm{b}} \\
1,2,3^{\mathrm{b}} \\
1,2,3^{\mathrm{b}}\end{array}$ \\
\hline A. rufescens & $\begin{array}{l}\text { Leiden } \\
\text { Wervicq-sud }\end{array}$ & $\begin{array}{l}\text { The Netherlands } \\
\text { France }\end{array}$ & $\begin{array}{l}13-9 \\
10-10\end{array}$ & $\begin{array}{l}1 \\
0\end{array}$ & $-1^{\mathrm{b}}$ \\
\hline A. citri & $\begin{array}{l}\text { Brazzaville } \\
\text { False Bay }\end{array}$ & $\begin{array}{l}\text { Congo } \\
\text { South Africa }\end{array}$ & $\begin{array}{l}7-10 \\
8-8\end{array}$ & $\begin{array}{l}0 \\
0\end{array}$ & - \\
\hline A. persimilis & $\begin{array}{l}\text { Brisbane } \\
\text { Sydney } \\
\text { Adelaide } \\
\text { Melbourne }\end{array}$ & $\begin{array}{l}\text { Australia } \\
\text { Australia } \\
\text { Australia } \\
\text { Australia }\end{array}$ & $\begin{array}{l}6-7 \\
5-6 \\
6-6 \\
6-6\end{array}$ & $\begin{array}{l}0 \\
0 \\
0 \\
0\end{array}$ & $\frac{-}{-}$ \\
\hline A. sp & Concord & United States & $10-12$ & 0 & - \\
\hline
\end{tabular}

a Wolbachia strains wAtab1, wAtab2 and wAtab3 are here named 1, 2 and 3, respectively.

bIn these lines, PCR products of the wsp gene have been sequenced and compared with already available sequences (Vavre et al, 1999). For the three Wolbachia strains, wAtab1, wAtab2 and wAtab3, no variation has been observed (100\% of homology). 
were used to amplify wasp nuclear DNA as a positive control for template DNA quality (see primers in Allemand et al, 2002). All extracts tested negative for this ITS-specific PCR were excluded from the analysis. DNA extraction and PCR amplification were performed as described in Dedeine et al (2001).

Diagnostic PCR assay was also used for specific detection of the three Wolbachia strains previously described in A. tabida (Vavre et al, 1999). Based on the Wolbachia wsp gene sequence of the three bacteria (Genbank accession numbers AF124856, AF124857, AF124859), specific primers were used for each of them (see Dedeine et al (2004) for sequences of primers). All three specific PCR assays were performed as separate reactions on DNA isolated from a single wasp. PCR conditions were as described in Vavre et al (2001), except that the annealing temperature used to detect wAtab3 was elevated to $55^{\circ} \mathrm{C}$.

A subset of PCR amplification products (one or two extracts per insect line) were directly purified and sequenced. PCR amplicons were gel-purified using Qiagen gel extraction spin columns and used directly in standard fluorescent cycle-sequencing PCR reactions using the Wolbachia strain-specific set of wsp gene primers. Sequencing reactions were cleaned and run on an automated ABI Prism 310 sequencer. Sequences were aligned to the Wolbachia sequences already available in A. tabida (Vavre et al, 1999).

\section{Obtention of aposymbiotic females and measure of oocyte production}

Aposymbiotic females were obtained by antibiotic treatment as described in Dedeine et al (2001). The antibiotic (rifampicin, Hoechst) was applied to the wasps through the developing Drosophila host larva: $150 \mu \mathrm{l}$ of a $2 \%$ rifampicin solution was added to $1.5 \mathrm{~g}$ of standard diet $(2 \mathrm{mg} / \mathrm{g})$. Treated Drosophila larvae transfer antibiotic to endoparasitic wasp larvae, which then develop into aposymbiotic adult wasps. To measure the number of oocytes produced, newly emerged females were fed honey for 5 days and then dissected in a phosphatebuffered saline solution $(1 \times$ PBS, pH 7.4). One ovary was transferred into a neutral red solution for $5 \mathrm{~min}$ and gently crushed between a slide and coverglass to disperse its contents. Oocytes were counted using a video system. Individual oocyte production was estimated as twice the number of oocytes in one ovary.

\section{Measurement of oocytes}

One ovary of females conditioned as described above was transferred on a slide in a phosphate-buffered saline solution $(1 \times$ PBS, $\mathrm{pH}$ 7.4). To avoid any deformation of oocytes, ovaries were neither covered with coverglass nor coloured. Preparations were observed under a light microscope (Zeiss, Germany), and the length $(L)$ and width $(W)$ of oocytes were measured using a digital video system. The volume of oocytes was estimated as the volume of a regular ellipsoid $\left(\pi / 3 L W^{2}\right)$.

\section{Developmental assay}

Developmental success of parasitoids was measured as the total number of offspring produced by eight 5-dayold fertilized females provided for $48 \mathrm{~h}$ with about $200 D$. melanogaster host larvae (24-h-old when parasitoids were introduced), in vials containing about $10 \mathrm{~g}$ of standard diet. Adult wasps normally emerged between 26 and 30 days after oviposition at $20^{\circ} \mathrm{C}$, males emerging before females. When all emerging wasps were counted, the remaining full Drosophila puparia were dissected to detect and count parasitoid larvae that died during development. Three environmental factors that can potentially affect the developmental success of parasitoids were studied: (1) the Drosophila host species. Four species were tested: D. melanogaster, D. subobscura, D. simulans and D. hydei), (2) the temperature of development $\left(15\right.$ and $20^{\circ} \mathrm{C}$ ) and (3) the richness of Drosophila diet: two diets were tested: one in which the energetic content was normal, the other in which it was reduced to $25 \%$.

\section{Measurement of parasitoid larvae}

At the time of host pupation, the parasitoid larva leaves the host body and stays in the puparium, thus becoming an ectoparasite (Carton et al, 1986). At this stage, Asobara larvae are easily visible through the Drosophila puparium. Batches were prepared with about 100 Drosophila eggs $(0-8 \mathrm{~h}$ old) in vials containing standard diet. After $48 \mathrm{~h}$ incubation, eight 5-days-old fertilized parasitoid females were added to each vial and allowed to oviposit for $8 \mathrm{~h}$ into Drosophila hosts. At 1, 4 and 7 days ( $\pm 3 \mathrm{~h}$ ) after Drosophila pupation, samples of parasitized Drosophila were dissected and parasitoid larvae were collected and measured ( $L$ : length and $W$ : width) using a micrometer. The volume of larvae was estimated as the volume of a regular ellipsoid $\left(\pi / 3 L W^{2}\right)$.

\section{Statistical analysis}

We conducted an ANOVA with one (infection status) or two (infection status, insect line) factors. The significance level $\alpha$ of ANOVAs, $P=0.05$, was adjusted following the Bonferroni procedure, to correct for multiple analyses (Sokal and Rohlf, 1995). Statistical analysis was performed using JMP ${ }^{\mathrm{TM}}$ version 5.1.1 (Software).

\section{Results}

\section{Wolbachia infection in the genus Asobara}

In $A$. tabida, all individuals proved co-infected by the three Wolbachia strains wAtab1, wAtab2 and wAtab3, and no variation was observed in the wsp sequences of Wolbachia strains from different insect lines (Table 1). Among the four other Asobara species surveyed, three proved uninfected ( $A$. citri, $A$. persimilis and $A$. $s p$ ). One population of $A$. rufescens (Wervicq-sud) was uninfected, while all individuals from the other population (Leiden) were infected (Table 1). Diagnostic PCR assay and sequencing analyses both showed that the infected $A$. rufescens population only harbours a single Wolbachia strain that is $100 \%$ homologous (based on 378 base pairs of wsp gene) to the strain wAtab1 infecting $A$. tabida (gene bank access: AF124856).

\section{Oocyte production in symbiotic and aposymbiotic A. tabida females}

The efficiency of antibiotic treatment was checked by testing at least 10 females per line for the presence of Wolbachia (217 females in total). All treated females were PCR-negative for the fts- $Z$ Wolbachia gene and PCR-positive for the ITS 2 region of the insect DNA. 
We compared oocyte production of symbiotic and aposymbiotic females from all $A$. tabida lines (Table 2). Interestingly, the statistical analysis performed only on symbiotic females shows that the oocyte production varies significantly between the $A$. tabida lines $(F=14.488 ; \mathrm{df}=15,225 ; P<0.0001)$. Consistent with previous studies (Dedeine et al, 2001, 2004), ovaries of aposymbiotic females were found completely empty of any oocyte in 13 European lines, indicating their total dependence on Wolbachia for oogenesis. Conversely, aposymbiotic females from Saanich, Seattle and Leiden lines were able to mature oocytes (Table 2). Comparing these three insect lines (two-way ANOVA) shows that (1) aposymbiotic females produced far fewer oocytes than symbiotic females $(F=1068.6 ; \mathrm{df}=1,110 ; P<0.0001)$ with $58.7 \%$ reduction in Saanich strain, $68.7 \%$ in Seattle and $80.4 \%$ in Leiden, and that (2) despite the lack of significance of the factor 'line' $(F=2.2 ; \mathrm{df}=1,110$; $P=0.114)$, the interaction between the two factors (infection status $\times$ line) is significant $(F=21.4 ; \mathrm{df}=1$, $110 ; P<0.0001)$, suggesting that the degree of dependence for oocyte production may be a continuous trait among $A$. tabida populations. Surprisingly, the three $A$. tabida lines able to produce a certain number of oocytes without Wolbachia are also the lines that produce on average the smaller number of oocytes when females are infected by Wolbachia (see Table 2).

\section{Oocyte size}

We compared the length and width of symbiotic and aposymbiotic oocytes (Table 3 ). In all cases, aposymbiotic

Table 2 Number of oocytes (mean \pm SD) produced by symbiotic and aposymbiotic females of the $16 \vec{A}$. tabida lines

\begin{tabular}{lcc}
\hline A. tabida line & Symbiotic & Aposymbiotic \\
\hline Villette & $294.6 \pm 20.4(13)$ & $0(13)$ \\
Kos & $291.4 \pm 15.3(14)$ & $0(14)$ \\
Gazeran & $288.7 \pm 23.7(14)$ & $0(14)$ \\
St Foy-lès-Lyon & $286.0 \pm 24.2(15)$ & $0(15)$ \\
Malaucène & $283.4 \pm 21.1(16)$ & $0(16)$ \\
Villeneuve d'Ascq & $283.3 \pm 20.9(15)$ & $0(15)$ \\
Vénérieu & $280.5 \pm 25.8(13)$ & $0(13)$ \\
St Laurent & $279.5 \pm 31.4(15)$ & $0(15)$ \\
Cordès & $276.3 \pm 30.9(14)$ & $0(14)$ \\
Lablachère & $275.4 \pm 29.5(15)$ & $0(14)$ \\
Wervicq-sud & $271.0 \pm 24.3(14)$ & $0(14)$ \\
Hoge veluwe & $267.5 \pm 24.3(14)$ & $0(12)$ \\
Pierrefeu & $267.3 \pm 23.2(20)$ & $0(20)$ \\
Leiden & $248.0 \pm 31.9(17)$ & $48.7 \pm 18.6(20)$ \\
Seattle & $242.1 \pm 21.9(15)$ & $75.8 \pm 20.5(20)$ \\
Saanich & $208.2 \pm 14.6(20)$ & $85.9 \pm 39.4(24)$ \\
\hline
\end{tabular}

Number of dissected females in brackets. oocytes are far smaller than symbiotic ones, both for width $(F=445.5 ; \mathrm{df}=1,594 ; P<0.0001)$ and length $(F=2637.9 ; \mathrm{df}=1,594 ; P<0.0001)$. The factor 'line' is also significant for width $(F=23.7 ; \mathrm{df}=2,594 ; P<0.0001)$ and interacts significantly with the factor infection status' $(F=20.703 ; \mathrm{df}=2,594 ; P<0.0001)$. Conversely, the factor 'line' is not significant for length $(F=1.6$; $\mathrm{df}=2$, 594; $P=0.205)$ and does not interact with the factor 'infection status' $(F=1.367 ; \mathrm{df}=2,594 ; P=0.255)$. It is clear, however, that most of the variation is attributable to infection status. Accordingly, reduction of oocyte volume ranges from $49.6 \%$ in Saanich strain and $53.7 \%$ in Seattle to $61.0 \%$ in Leiden.

\section{Developmental success}

To compare the developmental success of aposymbiotic and symbiotic individuals, we allowed aposymbiotic and symbiotic females to parasitize Drosophila larvae. Offspring emerged from each vial were counted 30 days after oviposition. While symbiotic females produced a substantial number of adult offspring, aposymbiotic females did not produce any. In vials where aposymbiotic females had oviposited, we could observe two clear morphological abnormalities of Drosophila puparia: (1) dark orange coloration instead of the yellow characterizing puparia containing Wolbachia-infected parasitoids, and (2) presence of an internal gas bubble. We then counted these easily distinguishable puparia in all vials (Table 4; in total $n=659)$. At dissection $(n=106)$, all puparia contained a single living parasitoid $A$. tabida larva, a gas bubble taking approximately one-third of the puparium volume and a liquefied mass of white host tissues. Finally, after 21 extra days, nothing emerged from the remaining 553 puparia, and dissection showed that the wasp larvae had died.

Table 3 Length, width and estimated volume of symbiotic (S) and aposymbiotic (A) oocytes from Saanich, Seattle and Leiden A. tabida females

\begin{tabular}{lcccc}
\hline Traits & $\begin{array}{c}\text { Infection } \\
\text { status }\end{array}$ & Saanich & Seattle & Leiden \\
& S & $0.238 \pm 0.006$ & $0.235 \pm 0.009$ & $0.237 \pm 0.008$ \\
Length $(\mathrm{mm})$ & $\mathrm{A}$ & $0.222 \pm 0.010$ & $0.222 \pm 0.010$ & $0.221 \pm 0.009$ \\
& & & & \\
Width $(\mathrm{mm})$ & $\mathrm{S}$ & $0.037 \pm 0.003$ & $0.036 \pm 0.003$ & $0.037 \pm 0.002$ \\
& $\mathrm{~A}$ & $0.027 \pm 0.003$ & $0.025 \pm 0.003$ & $0.024 \pm 0.002$ \\
Volume $\left(\mathrm{mm}^{3}\right)$ & $\mathrm{S}$ & $0.344 \pm 0.004$ & $0.325 \pm 0.004$ & $0.341 \pm 0.004$ \\
& $\mathrm{~A}$ & $0.174 \pm 0.004$ & $0.151 \pm 0.004$ & $0.132 \pm 0.004$
\end{tabular}

Each mean $( \pm S D)$ was calculated from the measure of 100 oocytes.

Table 4 Number of offspring and abnormal puparia (see text) produced (mean \pm SD) by symbiotic (S) or aposymbiotic (A) females

\begin{tabular}{|c|c|c|c|c|c|c|}
\hline \multirow[t]{3}{*}{ Infection status } & \multicolumn{6}{|c|}{ Offspring produced } \\
\hline & \multicolumn{2}{|c|}{ Saanich } & \multicolumn{2}{|c|}{ Seattle } & \multicolumn{2}{|c|}{ Leiden } \\
\hline & Adult & Abnormal puparia & Adult & Abnormal puparia & Adult & Abnormal puparia \\
\hline S & $86.8 \pm 14.7(15)$ & $0.0 \pm 0.0$ & $82.7 \pm 16.4(12)$ & $0.0 \pm 0.0(12)$ & $72.1 \pm 21.5(14)$ & $0.0 \pm 0.0(14)$ \\
\hline A & $0.0 \pm 0.0(24)$ & $14.5 \pm 11.1(24)$ & $0.0 \pm 0.0(20)$ & $8.1 \pm 8.0(20)$ & $0.0 \pm 0.0(22)$ & $6.8 \pm 9.3(22)$ \\
\hline
\end{tabular}

Number of vials studied in brackets. 
Since the developmental success of parasitoids may change according to complex interactions between the host species, temperature and feeding condition of host larvae (Vinson and Iwantsch, 1980; Carton et al, 1986; Wajnberg et al, 1990), we tested whether changing developmental conditions would allow aposymbiotic larvae to develop. In a series of similar experiments, we thus compared the developmental success of aposymbiotic and symbiotic $A$. tabida larvae under different rearing conditions (ie, we tested four different Drosophila species as developing host, two temperatures and two Drosophila diets), and clearly demonstrated that all aposymbiotic larvae failed to develop, although the presence of parasitoid larvae within abnormal host puparia (described above) demonstrated that wasps did lay eggs into Drosophila larvae (results not shown).

\section{Size and growth of parasitic larvae}

On days 1, 4 and 7 after Drosophila pupation, we compared the sizes of symbiotic and aposymbiotic wasp larvae after extraction from host puparia (Table 5). At 1 day after host pupation, aposymbiotic larvae are far smaller than symbiotic ones both in length $(F=324.3$; $\mathrm{df}=1,32 ; P<0.0001)$ and width $(F=96.0 ; \mathrm{df}=1,32$; $P<0.0001)$. Consequently, their volume is correspondingly reduced, showing their poor early development. Moreover, it is clear that symbiotic larvae go on growing till they have ingested all host Drosophila tissues (volumes increase by 3.5 between days 1 and 3, and by 1.2 between days 3 and 7; analysis of length: $F=70.732$; $\mathrm{df}=2,46 ; \quad P<0.0001$; analysis of width: $F=155.957$; $\mathrm{df}=2,46 ; P<0.0001)$. Conversely, aposymbiotic larvae do not grow further and remain unchanged over time both for length $(F=0.02 ; \mathrm{df}=2,440 ; P=0.98)$ and width $(F=0.12 ; \mathrm{df}=2,44 ; P=0.89)$.

\section{Discussion}

Many insect species depend on symbiotic microorganisms for essential physiological functions such as nutrition, reproduction or defense against predators or pathogens (Buchner, 1965; Margulis and Fester, 1991; Douglas, 1994). However, the evolutionary mechanisms by which such host functions have become dependent on the symbiont remain poorly understood. In this issue, the association between the parasitoid wasp A. tabida and Wolbachia may be an interesting model. Previous studies

Table 5 Length, width and estimated volume of symbiotic (S) and aposymbiotic (A) A. tabida larvae (line of Saanich) at three developmental stages: 1,3 and 7 days $( \pm 3 \mathrm{~h})$ after host pupation

\begin{tabular}{lcccc}
\hline Traits & $\begin{array}{c}\text { Infection } \\
\text { status }\end{array}$ & +1 day & +3 days & +7 days \\
\hline Length $(\mathrm{mm})$ & $\mathrm{S}$ & $1.738 \pm 0.112$ & $2.015 \pm 0.178$ & $2.211 \pm 0.096$ \\
& $\mathrm{~A}$ & $0.842 \pm 0.170$ & $0.854 \pm 0.165$ & $0.848 \pm 0.182$ \\
Width $(\mathrm{mm})$ & $\mathrm{S}$ & $0.555 \pm 0.018$ & $0.959 \pm 0.103$ & $1.006 \pm 0.096$ \\
& $\mathrm{~A}$ & $0.298 \pm 0.106$ & $0.304 \pm 0.115$ & $0.318 \pm 0.117$ \\
Volume $\left(\mathrm{mm}^{3}\right)$ & $\mathrm{S}$ & $0.560 \pm 0.072$ & $1.967 \pm 0.075$ & $2.367 \pm 0.075$ \\
& $\mathrm{~A}$ & $0.098 \pm 0.075$ & $0.105 \pm 0.075$ & $0.113 \pm 0.078$
\end{tabular}

Each mean $( \pm S D)$ was calculated from at least 15 larvae. have shown that among the three Wolbachia strains infecting each $A$. tabida host individual, one strain, wAtab3, is required for host oogenesis, whereas the two other strains, wAtab1 and wAtab2, do not have this function, but induce cytoplasmic incompatibility (Dedeine et al, 2001, 2004). This unique insect-bacteria association may document an example of evolutionary transition, where a parasite has become obligate for a single essential function of its host (Dedeine et al, 2001, 2003, 2004). In the present study, we report inter- and intraspecific variations in the presence and identity of Wolbachia, especially wAtab3, and on the role of infection on oocyte production by the host.

No Wolbachia infection has been detected in A. citri, A. persimilis, A. sp (North America) and in one population of $A$. rufescens, showing that these insects do not need Wolbachia to reproduce. Moreover, individuals of the infected $A$. rufescens population do not harbour wAtab3, as expected if they shared the same dependence on Wolbachia as $A$. tabida. In contrast, infected $A$. rufescens individuals harbour a Wolbachia strain, wAtab1, which also infects $A$. tabida, where it induces cytoplasmic incompatibility (Dedeine et al, 2004). These results suggest that the dependence on wAtab3 for oogenesis has evolved after the divergence of $A$. tabida from the other species studied here. Furthermore, the close relatedness of $A$. rufescens and A. tabida (Vet and Janse, 1984; Vet et al, 1984) suggests that the dependence on wAtab3 for oogenesis may be specific to A. tabida.

Within A. tabida species, individuals from all 16 geographical $A$. tabida lines tested here harbour the three Wolbachia strains, wAtab1, wAtab2 and wAtab3. Moreover, no variation was found in the wsp sequence within each Wolbachia strain between the different insect lines. Nevertheless, we found intraspecific variation in the degree of dependence of females on Wolbachia to produce oocytes: while aposymbiotic females cannot produce any oocyte in 13 European lines, aposymbiotic females from Saanich, Seattle and Leiden did produce a certain number of oocytes. However, these aposymbiotic females produced fewer and smaller oocytes than those produced by symbiotic females. Further, even though embryos are apparently able to hatch, the larvae produced die early during development. Thus in all cases, the dependence on Wolbachia infection for reproduction is complete in A. tabida, but, depending on their nuclear genotype, females lacking wAtab3 either fail to produce oocytes or produce few small oocytes that do not develop.

One question then is whether the two observed phenotypes (ie, no oocyte production and unviable larval offspring) have independent physiological bases, or whether they proceed from a unique deficiency of aposymbiotic females. Our results support the second hypothesis for two main reasons. First, aposymbiotic females produce far fewer oocytes than symbiotic ones (in all cases $<50 \%$ ), suggesting that, in addition to producing unviable offspring, they suffer strong inhibition of oogenesis. Second, during the curing process, triply infected females oviposit in Drosophila larvae which have been feeding on antibiotics. Thus, the $A$. tabida eggs were immersed in host body fluids that contain antibiotics once they had been injected into the host. It is likely that Wolbachia are eliminated at a very early developmental stage. However, the early removal 
of Wolbachia does not disturb further development of larvae, since we always obtain adult wasps of both sexes that look normal in all respects except for oocyte production (Dedeine et al, 2001). Thus, the two phenotypes observed are probably resulting from the same failure of aposymbiotic females to transmit a factor to their oocytes that is necessary for differentiation and development. Differences among $A$. tabida lines could simply result from some dosage effect.

One interesting result concerns the apparent relationship between the number of oocytes produced by infected females (their potential fecundity) and the number of oocytes produced by uninfected females (their degree of dependence on Wolbachia). Indeed, only females of the lines Saanich, Seattle and Leiden can produce some oocytes when uninfected. Unexpectedly, these lines also produce the fewest oocytes when infected. This suggests a genetic trade-off between the ability of females to produce some oocytes without Wolbachia, and their potential fecundity when infected, although additional quantitative genetic analyses are needed to better understand this relationship.

Another question is to understand how variation can be maintained within $A$. tabida species. Two main hypotheses have been proposed to explain how $A$. tabida became dependent on wAtab3 (reviewed in Dedeine et al, 2004). The hypothesis of the Sterility of Aposymbiotic Daughters (SAD) phenotype suggests that wAtab3 specifically sterilizes daughters that did not inherit wAtab3 from their infected mothers. As for cytoplasmic incompatibility, SAD-inducing Wolbachia would produce two molecules: a toxin produced in mothers but specifically inhibiting oogenesis of daughters, and a toxin-specific antidote produced in infected daughters only. According to the SAD hypothesis, differences between $A$. tabida lines that either completely or partly depend on wAtab3 to produce oocytes may be due either to differences in the efficacy of the toxic molecule produced by Wolbachia or to some resistance that insects have evolved against it.

Another possible hypothesis is that the high prevalence of wAtab3 within $A$. tabida populations protects nuclear genes that control oogenesis from selection, and could have allowed deleterious genetic changes (mutations and/or fixation of new allelic forms) to accumulate. According to this hypothesis, differences between $A$. tabida lines may be due to qualitative or quantitative differences in the genetic modifications that have accumulated. Lines from Saanich, Seattle and Leiden may have accumulated less genetic modification, or with less deleterious consequences, than the other lines. This outcome could be explained if these populations are in an evolutionary transitory phase and currently evolving towards complete dependence, or if some local specific selective pressures have partly purged them from deleterious genetic changes, either directly through selection or indirectly through pleiotropic effects. It is possible that the variation reported in this study may serve to distinguish the two hypotheses in future investigations.

In conclusion, this study raises many new questions, especially regarding the genetic basis of the poor performance of aposymbiotic females, the pathway of bacterial restoration and the way wAtab3 Wolbachia acquired this capacity. For all these questions, the reported intraspecific variation in the degree to which A. tabida females are dependent on wAtab3 to produce their oocytes will certainly provide a useful foundation for future genetic investigations.

\section{Acknowledgements}

We are most grateful to Jacques van Alphen and Patrice Eslin for providing Asobara strains. We also thank Drude Molbo, Michael E Ahrens, D DeWayne Shoemaker and two anonymous reviewers for their helpful comments on the manuscript. This work was partly supported by the Centre National de la Recherche Scientifique (UMR 5558).

\section{References}

Allemand R, Lemaitre C, Frey F, Boulétreau M, Vavre F, Nordlander G et al (2002). Phylogeny of six African Leptopilina species (Hymenoptera: Cynipoidea, Figitidae), parasitoids of Drosophila, with description of three new species. Ann Soc Entomol Fr 38: 319-332.

Bandi C, Anderson TJC, Genchi C, Blaxter ML (1998). Phylogeny of Wolbachia in filarial nematodes. Proc $R$ Soc Lond B 265: 2407-2413.

Bordenstein SR (2003). Symbiosis and the origin of species. In: Bourtzis K, Miller T (eds) Insect Symbiosis. CRC Press: LLC. pp 284-304.

Buchner P (1965). Endosymbiosis of Animals with Plant Microorganisms. Interscience: New York.

Carton Y, Boulétreau M, Van Alphen JJM, Van Lenteren JC (1986). The Drosophila parasitic wasps. In: Ashburner M, Carson HL, Thompson JN (eds) The Genetics and Biology of Drosophila. Academic Press: London. pp 347-394.

David JR (1962). A new medium for rearing Drosophila in axenic condition. Drosophila Info Serv 36: 128.

Dedeine F, Bandi C, Boulétreau M, Kramer LH (2003). Insights into Wolbachia obligatory symbiosis. In: Bourtzis K, Miller T (eds) Insect Symbiosis. CRC Press: LLC. pp 267-282.

Dedeine F, Vavre F, Fleury F, Loppin B, Hochberg ME, Boulétreau M (2001). Removing symbiotic Wolbachia bacteria specifically inhibits oogenesis in a parasitic wasp. Proc Natl Acad Sci USA 98: 6247-6252.

Dedeine F, Vavre F, Shoemaker DD, Boulétreau M (2004). Intraindividual coexistence of a Wolbachia strain required for host oögenesis with two strains inducing cytoplasmic incompatibility in the wasp Asobara tabida. Evolution 58: 2167-2174.

Douglas AE (1994). Symbiotic Interactions. Oxford University Press: New York.

Gottlieb Y, Zchori-Fein E (2001). Irreversible thelytokous reproduction in Muscidifurax uniraptor. Entomol Exp Applic 100: $271-278$.

Margulis L, Fester R (1991). Symbiosis as a Source of Evolutionary Innovation. The MIT Press: Cambridge, MA.

Rigaud T (1997). Inherited microorganisms and sex determination of arthropod hosts. In: O'Neill SL, Hoffmann AA, Werren JH (eds) Influential Passengers: Inherited Microorganisms and Arthropod Reproduction. Oxford University Press: New York. pp 81-101.

Sokal RR, Rohlf FJ (1995). Biometry. WH Freeman: New York.

Stouthamer R, Breeuwer JAJ, Hurst GDD (1999). Wolbachia pipientis: microbial manipulator of arthropod reproduction. Annu Rev Microbiol 53: 71-102.

Vavre F, Dedeine F, Quillon M, Fouillet P, Fleury F, Boulétreau M (2001). Within-species diversity of Wolbachia-induced cytoplasmic incompatibility in haplodiploid insects. Evolution 55: 1710-1714.

Vavre F, Fleury F, Lepetit D, Fouillet P, Boulétreau M (1999). Phylogenetic evidence for horizontal transmission of Wolba- 
chia in host-parasitoid associations. Mol Biol Evol 12: 1711-1723.

Vet LEM, Janse C (1984). Fitness of two sibling species of Asobara (Braconidae: Alysiinae), a larval parasitoid of Drosophilidae in different microhabitats. Ecol Ent 9: 345-354.

Vet LEM, Janse C, Van Achterberg C, Van Alphen JJM (1984). Microhabitat location and niche segregation in two sibling of drosophilid parasitoids: Asobara tabida (Nees) and A. rufescens (Foerster) (Braconidae: Alysiinae). Oecologia 61: 182-188.

Vinson SB, Iwantsch GF (1980). Host suitability for insect parasitoids. Annu Rev Entomol 25: 397-419.

Wajnberg E, Boulétreau M, Prévost G, Fouillet P (1990). Developmental relationships between Drosophila larvae and their endoparasitoid Leptopilina (Hymenoptera: Cynipidae) as affected by crowding. Arch Insect Biochem Physiol 13: 239-245.

Werren JH (1997). Biology of Wolbachia. Annu Rev Entomol 42: 587-609.

Werren JH, Windsor DM (2000). Wolbachia infection frequencies in insects: evidence of global equilibrium. Proc $R$ Soc Lond $B$ 262: 197-204.

Werren JH, Zhang W, Guo LR (1995). Evolution and phylogeny of Wolbachia: reproductive parasites of arthropods. Proc $R$ Soc Lond B 261: 55-71.

West SA, Cook J, Werren JH, Godfray HCJ (1998). Wolbachia in two insect host-parasitoid communities. Mol Ecol 7: $1457-1465$ 